\title{
Luis Estrada-González
}

\section{A BRIEF NOTE ON BÉZIAU'S 'RATHER TRIVIAL THEOREM" ABOUT LP}

\begin{abstract}
Béziau has recently argued that the logic LP commits dialetheists to trivialism and Martin has pointed out very clearly the main problems with that alleged result. My sole purpose here is to make the spirit of Martin's reply more concise, exhibiting as clearly as possible the logical defects in Béziau's reasoning. Additionally, I want to make some remarks on LP qua logic and not only as an interpreted language.
\end{abstract}

Keywords: dialetheia; paraconsistent logic; trivial interpretation

\section{Introduction}

Béziau has recently argued [see Béziau, 2016] that González-Asenjo's and Priest's logic LP commits the dialetheist to trivialism, the thesis that all propositions are true, and Martin [2018] has pointed out very clearly the main problems with Béziau's alleged result. My sole purpose here is to make the spirit of Martin's reply more concise - without drawing upon the notion of "truth simpliciter", that can do more harm than good, and pointing out that Béziau stuck unwarrantedly to one among the many possible LP-interpretations to fallaciously make his point. Additionally, I want to make some remarks taking into account that LP is a logic and not only an interpreted language.

These observations seem trivial to me, but I think it is necessary to state them again given the recent wave of attempts to warm up the war between the paraconsistent traditions, with talk about of the dialetheists' lies about the Liar [Arenhart and Melo, 2018], the antidialetheist evidence-based paraconsistent logic [Carnielli and Rodrigues, 2017], the incoherence of dialetheism [Arenhart and Melo, 2017], and 
so on. ${ }^{1}$ Dialetheism has several contentious elements, for sure, but the alleged triviality of LP is not to be counted among them.

\section{The very basics of $L P$}

González-Asenjo's/Priest's (zeroth-order) "logic of paradox", LP, can be defined over a language $L$ with a countable set of propositional variables and connectives $\{\sim, \wedge, \vee, \rightarrow\}$, with the usual formation rules. Let also $V=\{\top, *, \perp\}$ be a set of truth values, with the ordering $\perp<*<\top$, and $D^{+}=\{\top, *\}$ and $D^{-}=\{\perp\}$ as designated and antidesignated values, respectively. Consider a family of interpretations of $L$, that is, functions $\sigma: L \longrightarrow V$, with logical validity defined in the following way (where $\Gamma$ stands for a collection of formulas of $L$ ):

$\Gamma \models_{L P} A$ if and only if for each $\sigma$, if $\sigma(B) \in D^{+}$for any $B \in \Gamma$, then $\sigma(A) \in D^{+}$

and with the following truth tables:

\begin{tabular}{cc|c|c|c|c}
$A$ & $B$ & $\sim A$ & $A \wedge B$ & $A \vee B$ & $A \supset B$ \\
\hline$\top$ & $\top$ & $\perp$ & $\top$ & $\top$ & $\top$ \\
$\top$ & $*$ & $\perp$ & $*$ & $\top$ & $*$ \\
$\top$ & $\perp$ & $\perp$ & $\perp$ & $\top$ & $\perp$ \\
$*$ & $\top$ & $*$ & $*$ & $\top$ & $\top$ \\
$*$ & $*$ & $*$ & $*$ & $*$ & $*$ \\
$*$ & $\perp$ & $*$ & $\perp$ & $*$ & $*$ \\
$\perp$ & $\top$ & $\top$ & $\perp$ & $\top$ & $\top$ \\
$\perp$ & $*$ & $\top$ & $\perp$ & $*$ & $\top$ \\
$\perp$ & $\perp$ & $\top$ & $\perp$ & $\perp$ & $\top$
\end{tabular}

The properties of LP have been extensively studied in several places and are well-known; all the properties relevant to Béziau's claim are already in the 1979 paper by Priest; a reader with less spare time can simply check the first two sections of [Priest, 2006].

\section{An unfortunate rather trivial theorem (or two)}

Béziau wanted to prove a "rather trivial theorem" about LP to substantiate his claims that "The paraconsistent logic LP [...] can only be

1 More arguments in this vicinity against dialetheism can be found in [Tkaczyk, 2016], which are also discussed by Martin [2018]. 
supported by trivial dialetheists, i.e., those who believe that all sentences are dialetheias" or "LP is not compatible with relative dialetheism [the idea that only some propositions are dialetheias]". The theorem is this (I have adjusted the notation):

Theorem 1. In LP calling "true" both designated values, every molecular formula is a dialetheia.

Proof. Consider a molecular formula $M$. There is at least one distribution of truth-values giving to all its atomic formulas the value $*$. The unique extension of this distribution to a valuation obviously gives the value $*$ to $M$ and also to $\sim M$.

As a corollary, we see that there are not antilogies in LP, i.e., formulas which are always false, i.e., having always the value $\perp$.

[Béziau, 2016, p. 54]

But the proof does not deliver what is stated as a theorem. According to the proof, there is an interpretation, call it $\sigma^{T}$, such that for every atomic formula $p, \sigma^{T}(p)=*$ and, by the truth tables above, the only extension of this interpretation to all formulas gives $\sigma^{T^{+}}(A)=*$ for every formula $A$. Therefore, in such an interpretation, every formula is a dialetheia. But this is a trivial theorem in the worst sense: one does not get the result that "in the Logic of Paradox, LP, [...] all formulas are dialetheias", but only that "There are some LP-interpretations where all formulas are dialetheias". That is, one can get at most that in LP all formulas could be dialetheias. And this has been a well-known fact for a long time now. ${ }^{2}$

\section{One more}

More importantly, should we accept Béziau's reasoning, a considerable bunch of paraconsistent logicians from all the three great traditions would be trivialists at bottom. Consider this other "rather trivial theorem" analogous to Béziau's:

${ }^{2}$ Let $K_{3}$ be as LP but only with $\top$ as designated value, and $*$ and $\perp$ as antidesignated. Let an analetheia be a sentence that is neither $T$ nor $\perp[\mathrm{cf}$. Beall and Ripley, 2004]. Call logically false a sentence that is not designated. Exercise for the true aficionado: Prove by Béziau's method the rather trivial theorem that $K_{3}$ can be supported only by trivial analetheists, i.e., those who believe that all sentences are analetheias, or by logical nihilists, those who believe that all sentences are logically false. As a corollary, obtain that there are not tautologies in $K_{3}$, i.e., formulas which are always true, i.e., having always the value $T$. 
Theorem 2. In positive classical logic calling "true" the designated value in its usual two-valued semantics, every formula is true.

Proof. Positive classical logic is the negation-free fragment of classical logic. Consider a molecular formula $M$. There is at least one distribution of truth values giving to all its atomic formulas the value $T$. The unique extension of this distribution to a valuation obviously gives the value $T$ to $M$.

Therefore, positive classical logic can be supported only by trivialists, i.e., those who believe that all sentences are true. Hence, those who build and support paraconsistent logics on top of that are trivialists. I am sure that Béziau and other admirers of da Costa's $C$ logics and the LFI's would have some qualms about such a claim, and some Belgians could retort as well. And all of them would complain with good reason, since the proof does not deliver what the "theorem" states.

\section{Another unfortunate rather trivial theorem}

Hence, the claim in the abstract of Béziau's paper that "The paraconsistent logic LP [...] can only be supported by trivial dialetheists, i.e., those who believe that all sentences are dialetheias" is unwarranted. There is no proof that LP-dialetheists have to stick to that very interpretation in order to be LP-dialetheists. If Béziau wanted to say that LP-dialetheists do not have the logical means to get rid of that interpretation, that is, that they do not have the means to declare some formulas as logically false and false only, that is right, but that is quite a different story. Then, the "corollary" that LP does not have antilogies, i.e., formulas that always get the value false (and false only), is right, as it has been known almost since the first appearance of LP. But that still does not mean that LP-dialetheists do not have any means to obtain (just) false propositions; it is only that those means are extra-logical. This is just as classical and other logicians have to draw upon extra-logical resources to establish the truth or falsity of all atomic propositions, and hence of some molecular propositions as well. The LP-dialetheist has to find out among all the possible interpretations, not by pure logic alone, which ones correspond to reality. But the same happens with the classical and other logicians when they want to make claims about reality. Deciding beforehand that LP-dialetheists are committed to one and the 
worst possible interpretation is unwarranted and not fair to them. As I have said, this has been known for a long while now, but the reader will benefit from consulting the most recent and detailed expression of the idea, namely that to be found in [Martin, 2018, p. 213ff].

Thus, the claim that "LP is not compatible with relative dialetheism [the idea that only some propositions are dialetheias]" is unwarranted as well. Just for confirmation, consider another rather trivial theorem:

Theorem 3. In LP calling "true" both designated values, is compatible with relative dialetheism.

Proof. Consider a molecular formula $M$. Then, its atomic formulas might each get one of the three truth-values; exactly which ones depends on the distribution chosen. The value of that distribution extended into a valuation would obviously depend on the initial distribution chosen, and so $M$ might be just true and $\sim M$ just false, or the other way around, or both might be $*$. As a special case, consider the molecular formula $(p \wedge \sim p) \vee q$ and suppose $p$ is a dialetheia, i.e. $\sigma(p)=*$. Nonetheless, it is possible to have $\sigma(q)=\perp$ and then $\sigma((p \wedge \sim p) \vee q)=*$. Hence, even if both designated values are called 'true', there are molecular formulas that are designated and still not all the atomics need be designated. $\dashv$

Theorem 3, contradicting Theorem 1, states the fairly obvious claim that, in order to know a particular value for a formula in LP, one has to check the truth tables out, because contrary to what Béziau implies, there are several interpretations available and most of them do not make the language trivial, since there can be designated contradictions without the rest of formulas being designated. And, anticipating some cheap jokes, Theorem 3 and Theorem 1 are contradictories not because LP is the logic for trivial dialetheists, but simply because what is stated in Theorem 1 is wrong.

Although correct, Theorem 3 is unfortunate because, contrary to what it states, it sheds no (new) light at all on the most interesting philosophical applications of LP. It is just a grandiloquent way of exhibiting some features of the LP truth tables.

\section{LP is a logic}

Finally, if Theorem 1 is read as Béziau wants us to read it, it does not make sense of LP at all. LP, in being a logic and not only an interpretable 
language, is a collection of logically valid arguments, and it is well-known that LP is not trivial in the sense that not all arguments are valid in it. Were all formulas dialetheias in LP, every argument would be LP-valid, but that is not the case: LP is well-known for being paraconsistent and invalidating Explosion! ${ }^{3}$ This provides just more evidence that Béziau was unwarrantedly focusing on only one interpretation, without taking care of all of them, as it would be expected when doing logic. ${ }^{4}$

Had he paid more attention to the corollary of his "theorem", he would have found that LP does not have antilogisms, but this does not mean that all formulas are LP-theorems, since there are LP-contingencies as well, that is, formulas that under some valuations are designated and under some others are not designated. In fact, the logical truths of LP are exactly those of classical logic (as Béziau himself reports on [Béziau, 2016, p. 52]), and that means that the rest of formulas are not logical truths in LP. So the proof that LP drives the dialetheist into trivialism is a fantastic creature not to be found even in the paraconsistent jungle. ${ }^{5}$

Acknowledgments. Work supported by the PAPIIT project IN403719 "Intensionality all the way down: a new plan for logical relevance". A previous version of the note was presented at the Second ChinesePolish Workshop on Applied Logic (Torun, Poland, September 2018).

3 Again, this point has been known from the very first appearance of LP, and Martin [Martin, 2018, p. 215] also mentioned this in order to refute Béziau's claim that LP is a trivial logic.

4 And when doing logic, one can most of the time do without the trivial interpretation. It is hard to believe that one and the same person wrote "Trivial dialetheism and the Logic of Paradox" and the following remark on "What is paraconsistent logic?" [Béziau, 2000]:

The relation of consequence defined with a semantics in which there is the trivial model ( $w$ is model of all formulas) is the same as the one defined with this semantics minus the trivial model and vice-versa: if we add the trivial model to a semantics, it will not modify the induced consequence relation. Therefore we can suppose without loss of generality that the trivial model is never part of a semantics.

5 Nothing in the above implies that there could be no logics where all $C$-formulas, for a given class $C$ (be they atomic, molecular, all of them, etc.), are dialetheias. Examples of such abound, and Béziau mentions a number of them in his paper. My only claim here is about LP and its carrier set of formulas: it is simply not true that they are all dialetheias, although they can be so under the trivial interpretation, which is just one among many of the admissible interpretations. 
Thanks to Tomasz Jarmużek for discussion and the opportunity to visit Torun, and to Marek Nasienewski and Heinrich Wansing for useful discussion. Hitoshi Omori and Elisángela Ramírez-Cámara as well as to two anonymous referees deserve special thanks for further useful comments.

\section{References}

Arenhart, J.R. B, and E.S. Melo, 2017, "Is dialetheism self-coherent?", South American Journal of Logic 3 (1): 87-109.

Arenhart, J.R. B., and E.S. Melo, 2018, "Dialetheists' lies about the Liar", Principia 22 (1): 59-85.

Beal, J., and D. Ripley, 2004, "Analetheism and dialetheism", Analysis 64 (1): 30-35. DOI: 10.1093/analys/64.1.30

Béziau, J.-Y., 2000, "What is paraconsistent logic?", pages 95-111 in J. P. Van Bendegem, D. Batens, G. Priest and Ch. Mortensen (eds.), Frontiers of Paraconsistent Logic, Research Studies Press, Baldock.

Béziau, J.-Y., 2016, "Trivial dialetheism and the logic of paradox", Logic and Logical Philosophy 25 (1): 51-56. DOI: 10.12775/LLP.2015.022

Carnielli, W., and A. Rodrigues, 2017, "An epistemic approach to paraconsistency: A logic of evidence and truth", Synthese. DOI: 10.1007/s11229017-1621-7

Martin, B., 2018, "In defence of dialetheism: A reply to Beziau and Tkaczyk", Logic and Logical Philosophy 27 (2): 205-233. DOI: 10.12775/LLP. 2017. 030

Priest. G., 2006, In Contradiction, Clarendon Press, Oxford. DOI: 10.1093/ acprof : oso/9780199263301.001.0001

Tkaczyk, M., 2016, "The case of dialetheism", Logic and Logical Philosophy 25

(2): 203-224. DOI: $10.12775 /$ LLP. 2016.012

Luis EstradA-GONZÁLEZ

Institute for Philosophical Research

Graduate Program in Philosophy of Science

UNAM (Mexico)

loisayaxsegrob@gmail.com 